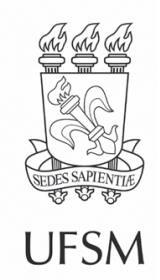

\title{
Artigos
}

\section{Estoque do potencial produtivo do Cerrado utilizando geotecnologias}

\author{
Potential wood stock in brazilian savanna using geotechnologies
}

\author{
Ivy Mayara Sanches de Oliveira' ${ }^{\bullet}$ \\ Aliny Aparecida dos Reis" ${ }^{\circ}$ \\ Eduarda Martiniano de Oliveira Silveira"II ๑ \\ Fausto Weimar Acerbi Júnior ${ }^{\prime v} \bullet$ \\ José Marcio de Mello'v $\bullet$ \\ 'Bracell São Paulo, Lençóis Paulista, SP, Brasil \\ "Universidade de Campinas, Campinas, SP, Brasil \\ I'University of Wisconsin-Madison, Madison, WI, Estados Unidos da América \\ IVUniversidade Federal de Lavras, Lavras, MG, Brasil
}

\section{RESUMO}

O monitoramento dos recursos naturais do nosso planeta é essencial para a obtenção de informações que possam subsidiar estratégias de conservação e utilização sustentável desses recursos. Tais estratégias se tornam ainda mais importantes em biomas ameaçados, como é o caso do Cerrado brasileiro, uma das savanas mais ricas e ameaçadas do mundo. Comumente o monitoramento das florestas é realizado por meio de inventários florestais, atividade onerosa, cara e extremamente difícil de ser realizada periodicamente em florestas extensas, como o Cerrado. Nesse sentido, o presente estudo teve como objetivo atualizar o mapeamento do estoque de volume e carbono em fragmentos de Cerrado, diminuindo a quantidade de levantamentos de campo por meio da aplicação de geotecnologias. Utilizaram-se dados de levantamento de campo de 61 fragmentos de Cerrado, sendo que em 25 deles foram realizadas 2 medições em um intervalo de 5 anos. Através dos dados de campo e valores de reflectância de imagens Landsat, modelos lineares múltiplos foram ajustados e validados. Posteriormente, esses modelos foram aplicados aos fragmentos que foram mensurados apenas uma vez. Dessa forma, foram obtidos os valores de volume e carbono para todos os 61 fragmentos, no ano da segunda medição, gerando mapas de produtividade atualizados.

Palavras-chave: Sensoriamento remoto; Inventário Florestal; Geoestatística 


\begin{abstract}
Monitoring natural resources of our planet is essential to gather information to support strategies for conservation and a sustainable use of these natural resources. This monitoring is even more important in endangered biomes, as is the case of Brazilian savannas, one of the most threatened and richest, in terms of biodiversity, savannas in the world. Usually, forest monitoring is accomplish by measuring the trees on the field, the so-called forest inventories, which are expensive, costly and extremely difficult to be performed periodically in extensive forests, as is the case of the Brazilian savannas. Therefore, this study aimed to update the information about volume and carbon stocks in savanna fragments in order to reduce tree measurements in the field. We used data from 61 savanna remnants, where 25 of them had 2 measurements with 5 years interval (monitoring). Multiple linear models, based on field data and reflectance values of Landsat images, were fitted and validated. Subsequently, the best models were applied to remnants that had only one measured, and then volume and carbon estimates were obtained for all 61 remnants in the second year of measurement. Additionally, maps updating the productivity of these remnants were generated.
\end{abstract}

Keywords: Remote sensing; Forest inventory; Geostatistics

\title{
1 INTRODUÇÃO
}

Devido à grande perda e degradação dos recursos naturais do nosso planeta, observamos que os acordos políticos para promover a conservação e o uso sustentável desses recursos têm ganhado destaque no cenário mundial. Contudo, para subsidiar tais políticas, é necessário o acesso a informações atuais sobre as florestas e biomas em escala espaço-temporal de maneira segura e confiável. Tais informações tornamse ainda mais necessárias em biomas ameaçados, como é o caso do Cerrado brasileiro. Por ser um hotspot mundial (KLINK; MACHADO, 2005) e se tratar de uma das savanas mais ricas em diversidade do mundo (MENDONÇA et al., 2008) as ciências florestais estão desenvolvendo diversos estudos com foco no Cerrado. Contudo, raros são os estudos relacionados à produção, seja em termos de volume de madeira ou de estoques de biomassa e de carbono (MIGUEL et al., 2015). Para Rezende et al. (2006), a escassez de informações em suas diferentes fitofisionomias está relacionada à grande heterogeneidade encontrada no bioma. Dessa forma, sendo o Cerrado um bioma ameaçado e seu potencial de produção pouco explorado, verifica-se a necessidade do aperfeiçoamento de técnicas para a obtenção de informações atualizadas e seguras para garantir a conservação e exploração sustentável de seus recursos. 
Tradicionalmente, o potencial produtivo das florestas é estimado por meio de amostragens, onde os dados são coletados por medições de campo que são dispendiosas e demoradas (HYYPPA et al., 2000) e muitas vezes, por falta de tempo e recursos financeiros, não são possíveis de serem realizadas em quantidade necessária de levantamentos terrestres, o que prejudica a confiabilidade dos resultados. Segundo Gómez et al. (2012), a avaliação da vegetação por meio de satélites, aliada aos inventários florestais, tem proporcionado observações rápidas, aumentando a precisão das estimativas do estoque da floresta e produzindo mapas confiáveis. Além disso, diversos estudos comprovam que as variáveis dendrométricas são espacialmente estruturadas (ALVARENGA et al., 2012; GUEDES et al., 2012). Dessa forma, a aplicação de técnicas geoestatísticas também é uma forma de tornar as estimativas mais acuradas em relação aos valores reais.

Sendo assim, o presente estudo teve como objetivo geral desenvolver uma metodologia que permita atualizar periodicamente o estoque de volume e carbono para fragmentos de Cerrado, visando diminuir a quantidade de levantamentos de campo, por meio da aplicação de geotecnologias. Os objetivos específicos foram (i) modelar e estimar o estoque de carbono e volume de madeira de fragmentos florestais, utilizando imagens de satélite aliadas a levantamentos de campo; (ii) aplicar os modelos em fragmentos sem remedição e gerar mapas de produtividade atualizados, por meio de interpoladores espaciais, para os estoques de carbono e volume.

\section{MATERIAL EMÉTODOS}

\section{1 Área de estudo}

O presente estudo foi realizado a partir de dados coletados em 1.553 parcelas inseridas dentro de 61 fragmentos de Cerrado distribuídos pelo estado de Minas Gerais (Figura 1), abrangendo as fitofisionomias do Campo Cerrado, Cerrado Sensu 
Stricto e Cerradão. Essas áreas fazem parte dos 165 fragmentos de vegetação nativa, mensurados no Inventário Florestal de Minas Gerais (SCOLFORO et al., 2008). O clima predominante é o tropical sazonal, de inverno seco, com temperatura média anual de $22,5^{\circ} \mathrm{C}$ e médias mensais muito variáveis. Pela classificação de Köppen, as áreas estudadas estão distribuídas nas regiões Cwa, Cwb, Aw e As (ALVARES et al., 2013).

Figura 1 - Localização dos fragmentos estudados

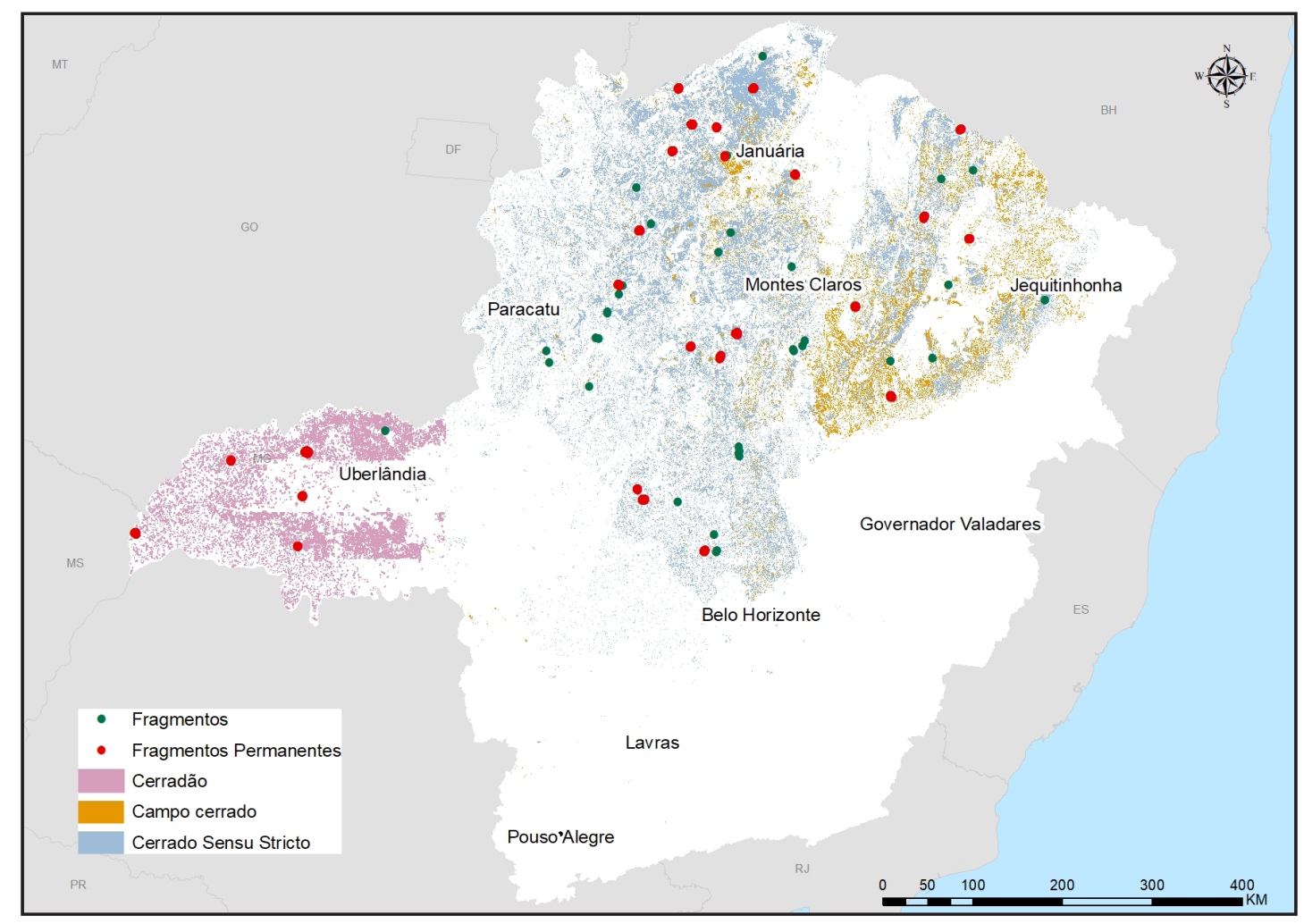

Fonte: Autores (2019)

As fitofisionomias do Cerrado apresentam um gradiente de vegetação com distintas características estruturais, as quais se expressam em parâmetros como altura média das árvores, densidade de indivíduos, porcentagem de cobertura do dossel, dentre outras (SOUZA; GALVÃO; SANTOS, 2009). Os fragmentos foram selecionados de forma a garantir que toda a variação da vegetação fosse amostrada. 


\subsection{Base de Dados}

No ano de 2005 (1 ${ }^{\text {a }}$ medição), foram alocadas, nesses fragmentos, 1.553 parcelas de $1.000 \mathrm{~m}^{2}(10 \times 100 \mathrm{~m})$, distribuídas de forma sistemática. Todas as árvores com diâmetro à altura do peito (DAP) $\geq 5 \mathrm{~cm}$ foram mensuradas. No ano de 2010 ( $2^{a}$ medição), foi realizada a remedição dessas árvores, contudo como se trata de uma atividade cara e demorada, o segundo levantamento ocorreu em apenas 25 desses fragmentos (647 parcelas), as quais compõem a chamada rede de fragmentos permanentes do Cerrado (MORAIS et al., 2013). Os valores de volume $\left(\mathrm{m}^{3}\right)$ e carbono (Mg), para todas as parcelas nos dois anos de medição, foram calculados por meio das equações ajustadas por Scolforo (2008).

Foram selecionadas 15 cenas do satélite Landsat 5 TM, no ano de 2010, de forma a cobrir todos os 61 fragmentos de Cerrado. As imagens foram adquiridas no site do United States Geological Survey - USGS (http://earthexplorer.usgs.gov), com os pré-processamentos a nível do subproduto Landsat Surface Reflectance, eliminando a necessidade de correções, uma vez que essas imagens já possuem correção geométrica e valores de reflectância a nível do solo. As datas das imagens foram: $21 / 04,09 / 05,16 / 05,28 / 05,10 / 06,17 / 06,24 / 06,15 / 07$ e 17/07, todas do ano de 2010. A escolha das datas das imagens se deu pela observação da inexistência de nuvens e/ou sombra de nuvens sobre o fragmento, respeitando uma proximidade entre as datas, para que correspondam a uma interpretação correta do estado de desenvolvimento da vegetação.

\subsection{Modelagem}

Visando atualizar a estimativa dos valores de volume (v2) e carbono (c2) de todos os 61 fragmentos do Cerrado para ano de 2010 ( $2^{a}$ medição), modelos de regressão foram ajustados por meio de dados dos 25 fragmentos remedidos. Considerou-se como preditoras, as variáveis dendrométricas obtidas a partir de dados mensurados 
em campo no ano de 2005 e valores de reflectância do ano em que se deseja obter as novas estimativas, no caso, o ano de 2010. A partir das coordenadas geográficas dos centroides das parcelas, extraíram-se os dados de reflectância, em seis bandas espectrais do sensor Thematic Mapper (TM) e de 5 índices de vegetação, sendo eles o índice de razão simples (SR), o índice de vegetação por diferença normalizada (NDVI), o índice de vegetação verde (VIg), o índice de área foliar (IAF) e o índice de vegetação ajustado ao solo (SAVI).

Inicialmente foi feita uma análise exploratória dos dados para entender seu comportamento geral. Posteriormente, os fragmentos foram separados por fitofisionomia, segundo metodologia utilizada por Scolforo et al. (2008). Foram ajustados modelos por fitofisionomia e característica estudada, totalizando seis modelos de regressão do tipo linear múltiplo. O procedimento utilizado no ajuste foi o "stepwise" de seleção de variáveis significativas. A multicolinearidade foi avaliada pelo teste de fator de inflação da variância ("Variance Inflation Factor", VIF). Das regressões ajustadas, foram analisados o coeficiente de determinação $\left(R^{2}\right)$, o erro padrão residual (Sxy) e o gráfico de dispersão dos resíduos. O poder de predição dos modelos foi avaliado a partir da técnica de validação preditiva (30\% dos dados), com base no coeficiente de determinação e na raiz do erro quadrático médio (RMSE). Após a validação, os modelos foram aplicados às parcelas dos 36 fragmentos sem remedição, obtendo-se assim os valores de volume (v2) e carbono (c2) para as parcelas que não estão sendo monitoradas. Dessa forma, todas as 1553 parcelas passaram a conter informações estimadas para o ano de 2010.

Os dados estimados para o ano de 2010 passaram por uma nova análise exploratória, para verificar sua estrutura espacial e tendência. Através do software R, foram ajustados semivariogramas experimentais para v2 e c2. Os parâmetros dos semivariogramas experimentais foram levados ao software ArcGis, onde foi efetuada a krigagem, interpolador geoestatístico que permite representar quantitativamente 
a variação de um fenômeno regionalizado no espaço (JOURNEL; HUIJBREGTS, 1978).

Dessa forma, a krigagem permitiu a obtenção de mapas de produtividade de volume ( $\mathrm{m}^{3} / \mathrm{ha}$ ) e carbono ( $\left.\mathrm{mg} / \mathrm{ha}\right)$ para o ano de 2010.

O fluxograma apresentado abaixo ilustra de forma prática as etapas realizadas

(Figura 2).

Figura 2 - Fluxograma

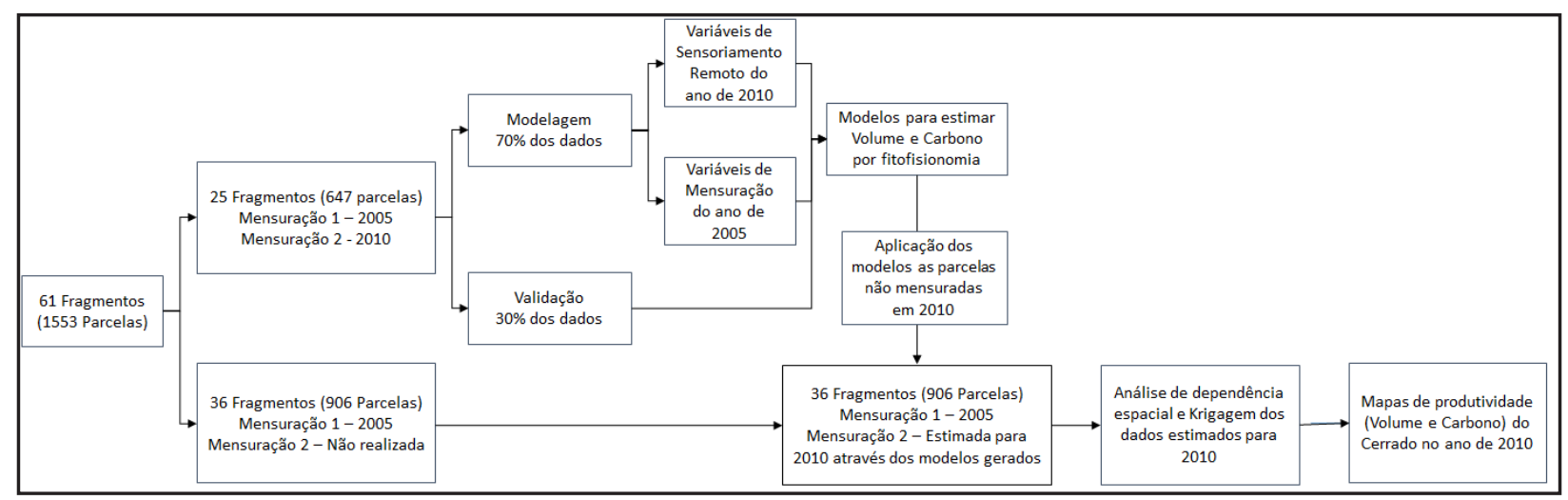

Fonte: Autores (2019)

\section{RESULTADOS EDISCUSSÃO}

Os modelos ajustados apresentaram bom desempenho quanto às estimativas de volume (v2) e carbono (c2), nas parcelas estudadas, com baixos valores para erro-padrão da estimativa e altos valores para coeficiente de determinação (Tabela 1).

Embora haja relação direta entre os valores de reflectância e a produção das florestas (SCHLERF; ATZBERGER; HILL, 2005), os altos valores de $\mathrm{R}^{2}$ foram influenciados pela seleção das variáveis dendrométricas obtidas durante o primeiro levantamento (v1 e c1). Isso pode ser comprovado quando se compara esse resultado com os encontrados em trabalhos que ajustaram modelos para predizer parâmetros florestais apenas por meio de dados de reflectância, como Santos et al. (2017) e Miguel et al. (2015). Dessa forma, sendo o Cerrado um bioma diverso, a seleção das variáveis 
dendrométricas para compor os modelos foi importante para balizar o resultado da estimativa, gerando modelos mais confiáveis e precisos.

Tabela 1 - Estimativas dos parâmetros dos modelos lineares múltiplos, com valores de coeficiente de determinação $\left(R^{2}\right)$ em percentagem e do erro padrão da estimativa na unidade da variável estimada (Sxy)

\begin{tabular}{|c|c|c|c|c|c|c|c|c|c|}
\hline \multirow{2}{*}{ Fitofisionomia } & \multirow{2}{*}{ Modelo } & \multirow{2}{*}{ Variável } & \multicolumn{7}{|c|}{ Parâmetros } \\
\hline & & & $\beta 0$ & $\beta 1$ & $\beta 2$ & $\beta 3$ & $\beta 4$ & $\mathbf{R}^{2}$ & Sxy \\
\hline \multirow{4}{*}{ Cerradão } & \multirow{2}{*}{ M1 } & \multirow{2}{*}{ Volume (v2) } & & v1 & TM3 & & & & \\
\hline & & & 4,354 & 0,921 & $-27,432$ & & & 0,910 & 1,262 \\
\hline & \multirow{2}{*}{ M2 } & \multirow{2}{*}{ Carbono (c2) } & & c1 & NDVI & & & & \\
\hline & & & 0,095 & 0,870 & 1,351 & & & 0,901 & 0,275 \\
\hline \multirow{4}{*}{ Campo Cerrado } & \multirow{2}{*}{ M3 } & \multirow{2}{*}{ Volume (v2) } & & v1 & SAVI & NDVI & Vlg & & \\
\hline & & & $-0,581$ & 1,097 & $-0,966$ & 2,705 & $-2,267$ & 0,986 & 0,202 \\
\hline & \multirow{2}{*}{ M4 } & \multirow{2}{*}{ Carbono (c2) } & & c1 & SAVI & NDVI & VIg & & \\
\hline & & & $-0,176$ & 1,105 & $-0,269$ & 0,778 & $-0,649$ & 0,987 & 0,058 \\
\hline \multirow{4}{*}{$\begin{array}{l}\text { Cerrado Sensu } \\
\text { Strictu }\end{array}$} & \multirow{2}{*}{ M5 } & \multirow{2}{*}{ Volume (v2) } & & v1 & IAF & TM4 & & & \\
\hline & & & 1,152 & 1,099 & 0,511 & $-5,420$ & & 0,961 & 0,577 \\
\hline & \multirow{2}{*}{ M6 } & \multirow{2}{*}{ Carbono (c2) } & & c1 & TM4 & IAF & TM3 & & \\
\hline & & & 0,263 & 1,097 & $-2,043$ & 0,263 & 2,409 & 0,963 & 0,167 \\
\hline
\end{tabular}

Fonte: Autores (2019)

A fitofisionomia Cerradão é caracterizada por apresentar dossel fechado e maiores valores de volume e área basal. As variáveis explicativas selecionadas, oriundas do sensoriamento remoto, para o Cerradão, foram: TM 3 para estimar volume e o NDVI para estimar o Carbono. A TM3 corresponde à banda do vermelho, região de alta absorção da clorofila, utilizada para caracterizar áreas de vegetação densa e uniforme. O índice NDVI geralmente apresenta alta correlação com as estruturas da paisagem e é derivado da razão ponderada das bandas TM3 e TM4, sendo a TM4 a região do infravermelho próximo, chamada de zona de alta reflectância da vegetação, apresentando alta sensibilidade às copas das florestas. A fitofisionomia campo cerrado apresenta baixo número de árvores e maior densidade de estrato herbáceo, apresentando, portanto, baixos valores de volume e área basal. Dessa forma, a associação das variáveis com os índices NDVI, SAVI e Vlg é plausível, uma vez que o NDVI 
é um indicador sensível da quantidade da vegetação, o SAVI minimiza interferência da reflectância do solo e o VIg é capaz de captar a presença de gramíneas e árvores decíduas, pois, em determinadas ocasiões, elas tornam a percepção do comprimento de onda do verde maior que o do vermelho. O Cerrado Sensu Stricto possui estrato arbóreo arbustivo apresentando valores medianos de volume de madeira. Para essa fitofisionomia, as variáveis preditoras que apresentaram eficácia nas estimativas foram TM3, TM4 e IAF. O índice de vegetação IAF é um subproduto do NDVI e SAVI e indica a biomassa de cada pixel da imagem, ou seja, avalia a biomassa por unidade de área.

Segundo os estudos de Berra et al. (2012), existe alta correlação positiva entre volume de madeira e os valores de NDVI e SR, para plantios de eucaliptos. Almeida et al. (2014) ajustaram modelos para estimar características dendrométricas da Caatinga a partir de dados do sensor TM do satélite Landsat 5. Os autores encontraram as maiores correlações entre o volume de madeira e os índices NDVI e SR. O coeficiente de determinação $R^{2}$ foi igual a 0,6 para o melhor modelo ajustado.

Figura 3 - Gráficos de resíduos dos modelos ajustados

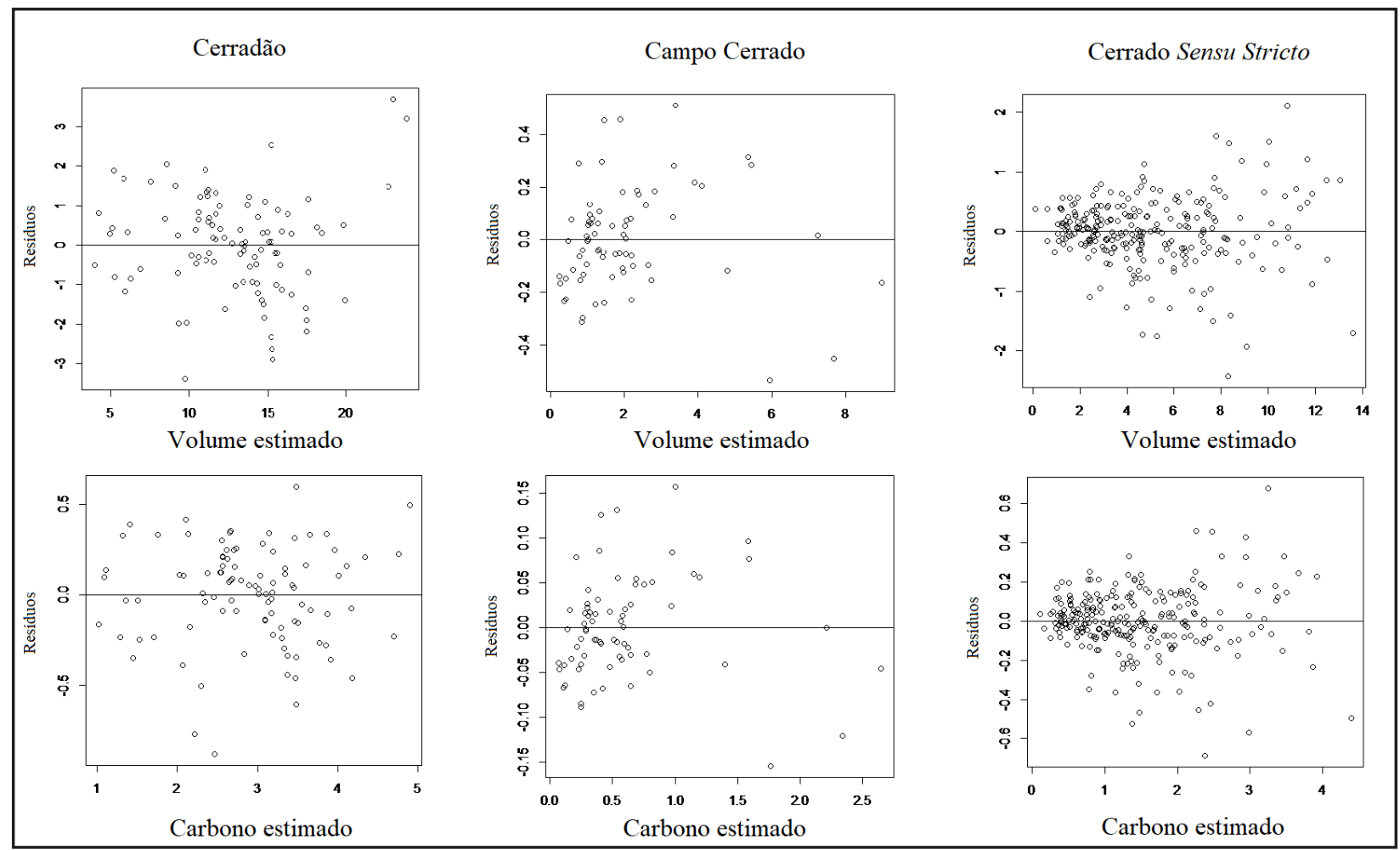

Fonte: Autores (2019) 
Todos os modelos ajustados apresentaram um $\mathrm{R}^{2}$ maior que 0,90, o que significa que conseguem explicar cerca de $90 \%$ da variação em volume e carbono. O teste VIF não apresentou multicolinearidade entre as variáveis explicativas (VIF<10). Na Figura 3, estão apresentados os gráficos de dispersão dos resíduos para cada um dos modelos ajustados. Os melhores ajustes ocorreram no Cerrado Sensu Stricto onde os resíduos estão melhor distribuídos em decorrência do maior número de fragmentos inventariados para essa fitofisionomia.

É importante considerar a complexidade das variáveis avaliadas, uma vez que as parcelas pertencem a fragmentos com diferentes graus de antropização, estágios sucessionais e árvores com portes diferentes, o que por si só já acarreta num aumento da variabilidade vegetacional (SCOLFORO; OLIVEIRA; CARVALHO, 2008). Na Figura 4, estão apresentados resultados da validação preditiva.

Figura 4 - Gráficos dos valores observados versus estimados com a dispersão dos valores preditos. Raiz quadrada do erro médio (RMSE\%) e coeficiente de determinação dos valores observados versus estimados ( $\left.\mathrm{R}^{2} \%\right)$, para os modelos ajustados

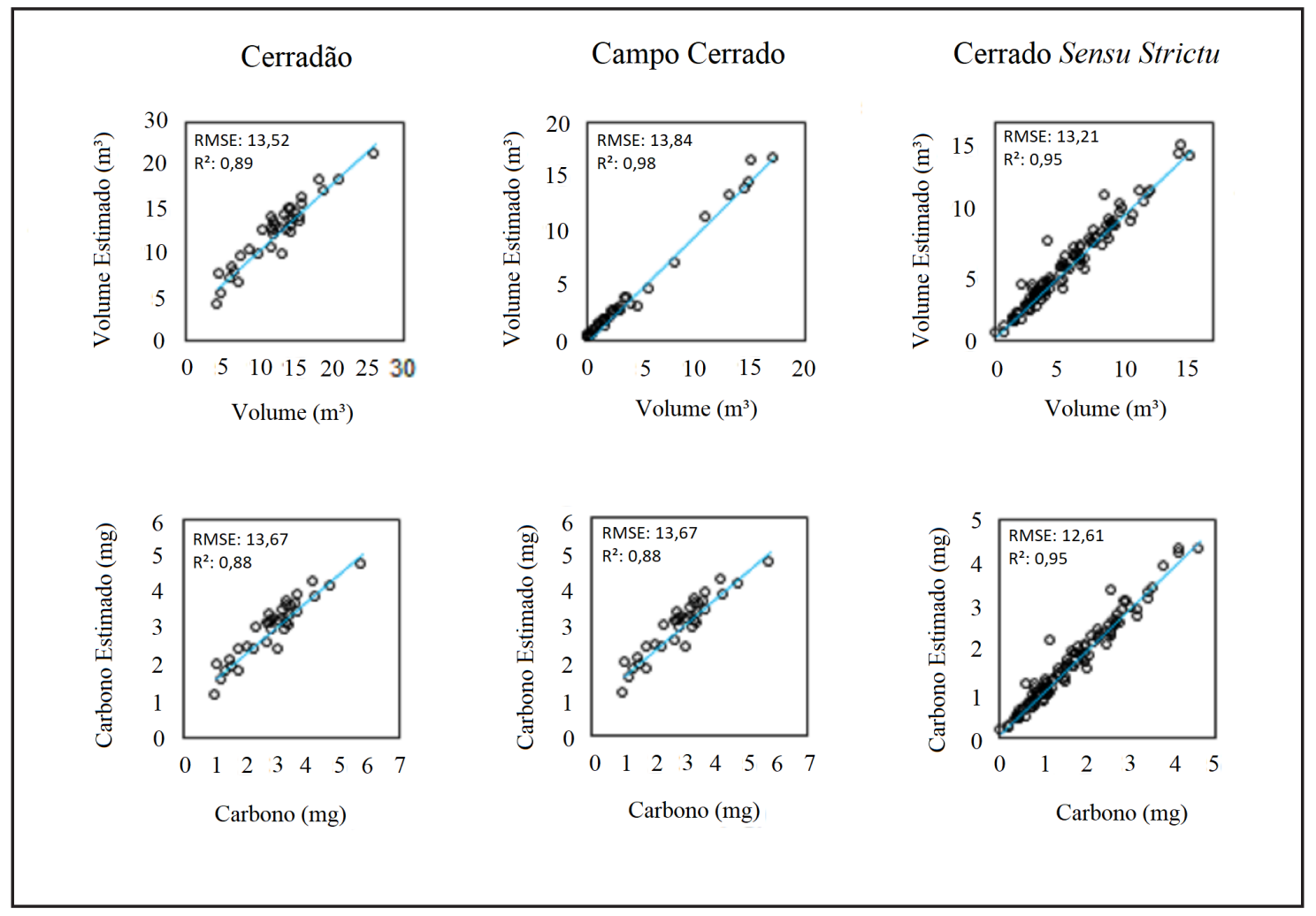

Fonte: Autores (2019) 
O teste de validação preditiva demonstra que os modelos se aderem muito bem às regiões que não fizeram parte do ajuste. Após a validação, os modelos foram aplicados às parcelas dos fragmentos que não foram remedidos no ano de 2010 . Dessa forma, obtivemos os valores de volume e carbono, para todas as 1.553 parcelas no ano de 2010. Esses dados foram posteriormente utilizados na interpolação das informações para todo o Cerrado. Para a geração de mapas contínuos de volume e carbono, semivariogramas experimentais (Tabela 2, Figura 5) foram ajustados para as duas características estudadas.

Tabela 2 - Parâmetros efeito pepita $\left(\tau^{2}\right)$, patamar $\left(\sigma^{2}\right)$ e alcance teórico $(\phi)$ para o modelo de função de semivariância selecionado para as variáveis estudadas

\begin{tabular}{lcccc}
\hline & Modelo & $\boldsymbol{\tau}^{\mathbf{2}}$ & $\boldsymbol{\sigma}^{\mathbf{2}}$ & $\boldsymbol{\phi}(\mathbf{m})$ \\
\hline Volume & Gaussiano & 6,20 & 9,2 & 2000,00 \\
Carbono & Esférico & 0,5 & 0,89 & 2249,60 \\
\hline
\end{tabular}

Fonte: Autores (2019)

Figura 5 - Semivariogramas experimental (pontos) e o modelo de melhor desempenho ajustado para volume (a) e carbono(b)

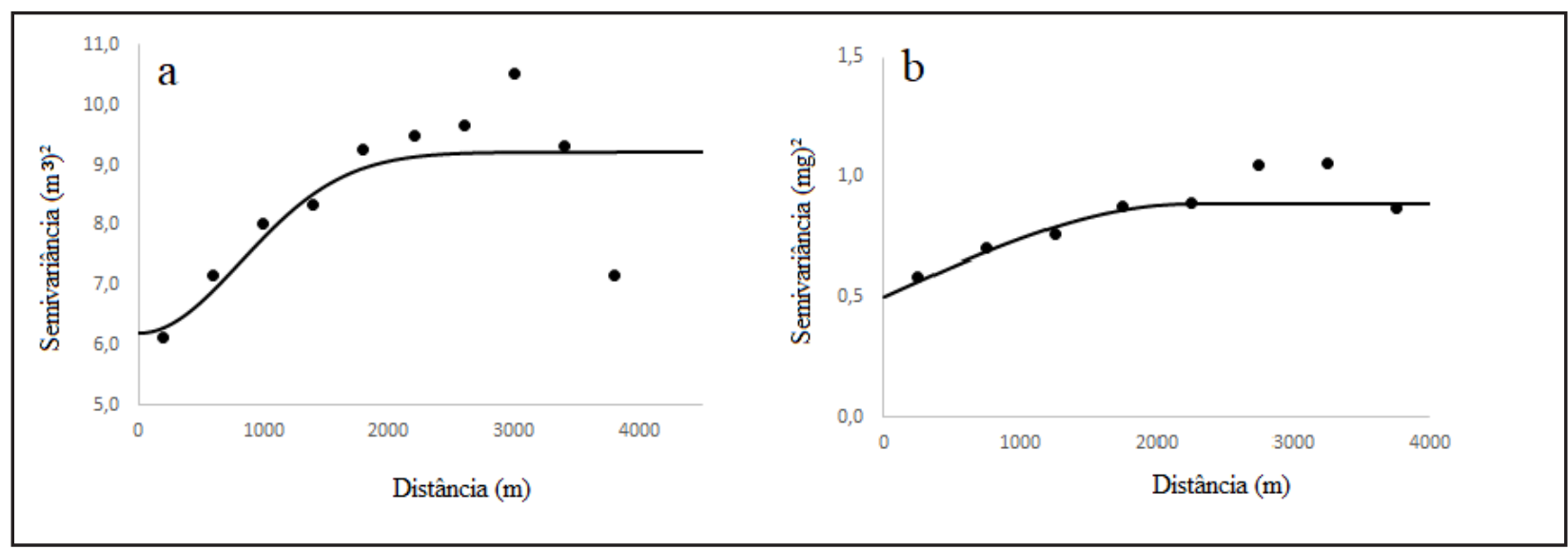

Fonte: Autores (2019) 
O modelo de melhor ajuste ao semivariograma experimental é influenciado pela natureza dos dados (ACERBI JúNIOR et al., 2015). O efeito pepita representa a variação dos dados que não foram explicadas pelo modelo espacial (YAMAMOTO; LANDIM, 2013). Dessa forma, quanto menor, mais desejável, garantido maior precisão da krigagem. O parâmetro alcance $(\phi)$ evidencia a magnitude da dependência espacial dos dados. O valor do alcance é alto em função das distâncias entre os pontos de amostragem que se localizam numa área extensa como a do estado de Minas Gerais. Após o ajuste, a interpolação dos dados foi realizada através da krigagem ordinária, interpolador geoestatístico, que leva em consideração a estrutura de dependência espacial das variáveis. Os mapas das Figuras 6 e 7 representam a produtividade do Cerrado, em termos de Volume $\left(\mathrm{m}^{3}\right)$ e Carbono (Mg), no ano de 2010.

Figura 6 - Mapeamento do estoque de volume $\left(\mathrm{m}^{3} / \mathrm{ha}\right)$ no Cerrado de Minas Gerais no ano de 2010

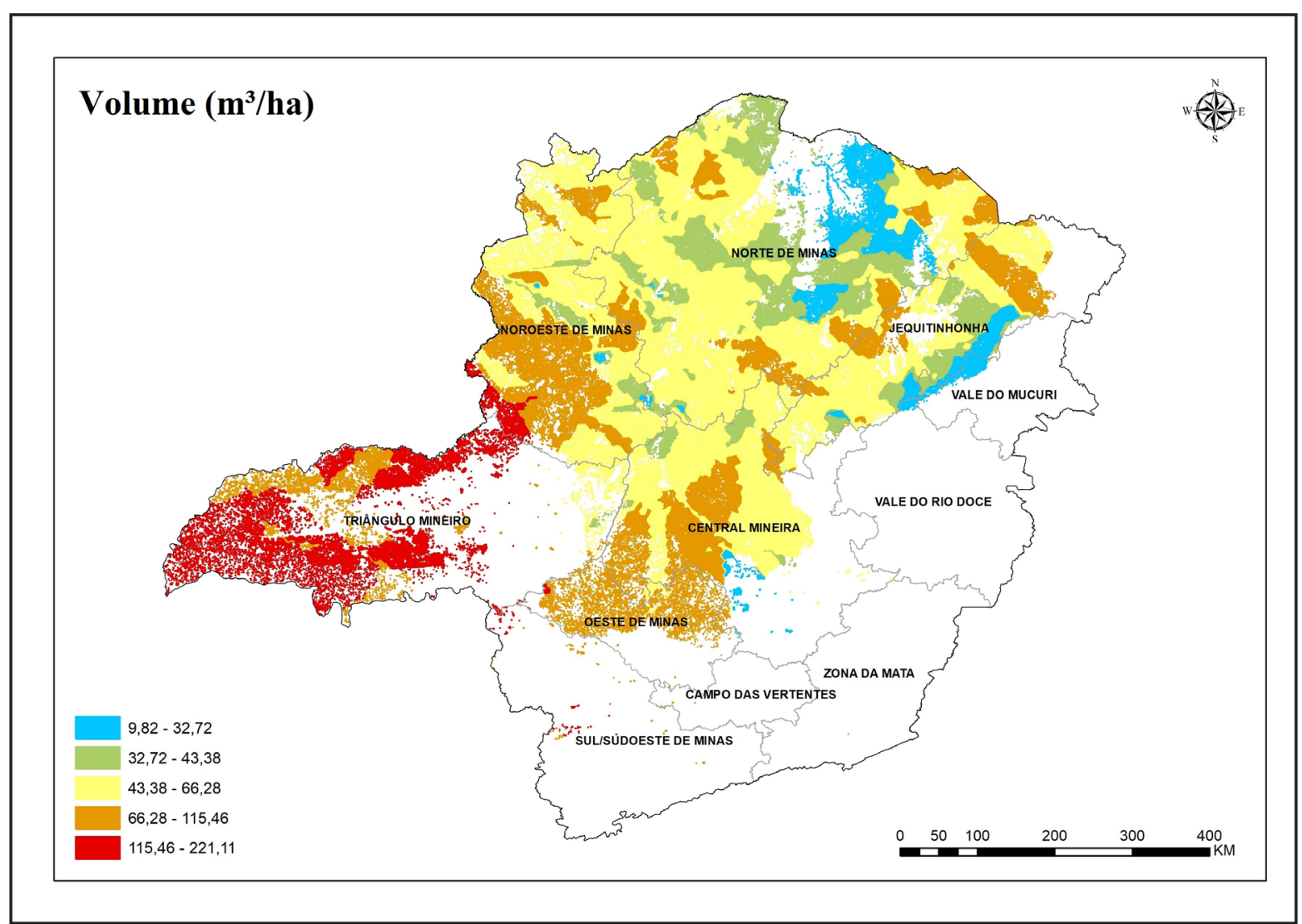

Fonte: Autores (2019) 
Os mapas dos estoques de volume e carbono demonstram o comportamento global dessas características no Cerrado em Minas Gerais, no ano de 2010. De maneira geral, observa-se que existe uma alta relação entre a distribuiç̧ão dos estoques de volume e carbono e a fitofisionomia em que se encontram. Isso se deve a diferenças em sua composição florística. Os maiores valores de estoque das variáveis estudadas ocorrem em maior concentração na região do Triângulo Mineiro, enquanto menores valores são observados mais ao Norte do estado. Esse comportamento já era esperado, visto que a vegetação ali presente é, em geral, mais densa e possui árvores com maiores dimensões, além de ciclagem de nutrientes mais intensa (WATZLAWICK et al., 2012).

Figura 7 - Mapeamento do estoque de carbono (Mg/ha) no Cerrado de Minas Gerais no ano de 2010

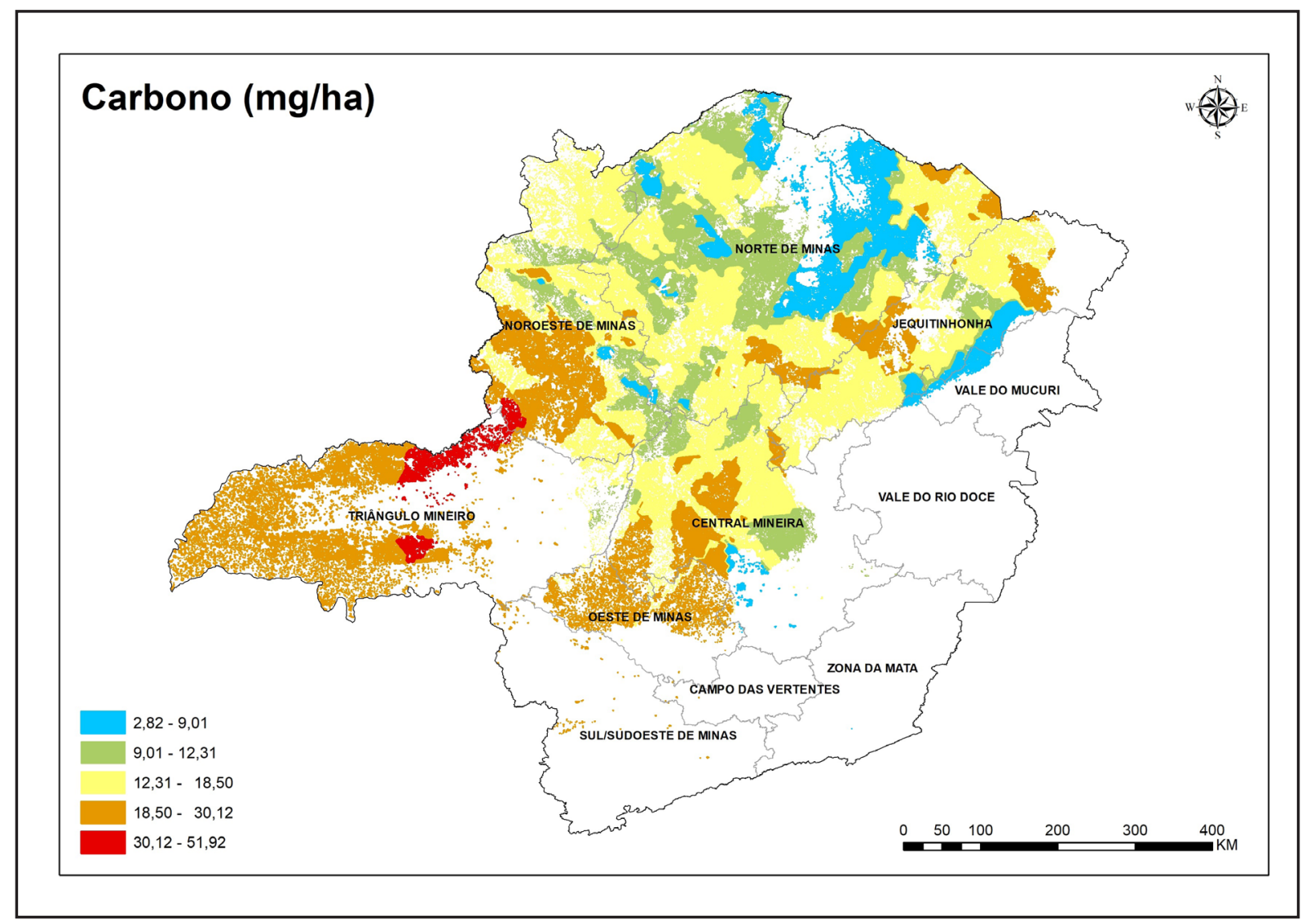

Fonte: Autores (2019) 
O campo cerrado apresenta maior ocorrência nas regiões do vale do Jequitinhonha e Norte do estado e observa-se que ali estão inseridas as classes de valores mais baixos das características avaliadas. Isso ocorre devido ao fato dessa fitofisionomia ser composta, segundo Carvalho et al. (2008), em sua maioria, por um estrato herbáceo, resultado da combinação do tipo de solo, altas temperaturas e baixa precipitação anual (850 mm). Dessa forma, era esperado que essa região apresentasse uma produtividade menor para as características avaliadas.

O Cerrado Sensu Stricto ocorre predominantemente de Norte a Sul na porção central do estado. Nessa região, ocorre maior variação de clima, tipo de solo e relevo, tais características influenciam a produção e o acúmulo de biomassa em áreas florestais, principalmente no caso de florestas nativas, resultando em diferenças nos estoques das florestas (AMARO et al., 2013). Conforme observado nos mapas de produtividade (Figuras 6 e 7), nesses locais, os valores de volume e carbono se mostraram bastante variados. Silva et al. (2014) mapearam a distribuição do volume de madeira para o Cerrado Sensu Stricto em Minas Gerais por meio de técnicas de krigagem. Nesse estudo, os autores observaram a existência de um gradiente decrescente de volume do centro do estado para a região Norte, predominando ao Norte uma volumetria inferior a 30 $\mathrm{m}^{3} /$ ha de madeira, que está ligada ao balanço hídrico da região. O Cerradão tem uma ocorrência maior no Sudoeste do estado, região do Triângulo Mineiro e é um tipo de savana florestada, apresentando dossel fechado e árvores mais grossas, sendo, portanto, uma fitofisionomia mais produtiva do que as anteriores, algo visivelmente observado nos mapeamentos gerados. Segundo Carvalho et al. (2008), essa região tem condições favoráveis para o desenvolvimento das plantas, como o calor, água e disponibilidade de nutrientes adequados.

Scolforo et al. (2015) mapearam a distribuição espacial do estoque de carbono, por meio de krigagem com regressão, nos diversos biomas de Minas Gerais, e verificaram que a maior concentração se encontra na região Norte do estado, local onde a vegetação nativa predominante é o Cerrado. Os autores encontraram valores 
médios de carbono variando de 8,2 a $26,6 \mathrm{mg} / \mathrm{ha}$ ao longo de todo o Cerrado. Morais et al. (2014) quantificaram e espacializaram o estoque de carbono da serrapilheira do cerrado de Minas Gerais utilizando krigagem e concluíram que a fitofisionomia Campo Cerrado tem o mais baixo estoque e o Cerradão o mais alto. Santos et al. (2017) estimaram e espacializaram diversos parâmetros florestais, por meio de técnicas de sensoriamento remoto em uma área de Cerrado. Este estudo comprovou a capacidade de modelos gerados, por meio de dados de sensoriamento remoto, captarem áreas com descontinuidade de vegetação, apresentando, nessas regiões, baixos valores de área basal. A Tabela 3 apresenta os valores médios das características avaliadas para as diferentes fitofisionomias inseridas no Cerrado, para o ano de 2010 com base nas krigagens realizadas.

Tabela 3 - Valores médios de volume e carbono por hectare para as fitofisionomias do Cerrado no ano 2010

\begin{tabular}{lccc}
\hline & Campo Cerrado & Cerrado Sensu Stricto & Cerradão \\
\hline Volume $\left(\mathrm{m}^{3} / \mathrm{ha}\right)$ & 47,02 & 50,18 & 140,82 \\
Carbono $(\mathrm{mg} / \mathrm{ha})$ & 15,39 & 14,99 & 34,79 \\
\hline
\end{tabular}

Fonte: Autores (2019)

Os valores médios variaram pouco em relação às médias encontradas por Scolforo et al. (2008), por meio de dados mensurados em campo no ano de 2005 para esses mesmos locais. Os valores e tendências encontradas também se assemelham aos encontrados nos estudos supracitados (SILVA et al., 2014; SCOLFORO et al., 2015; SANTOS et al., 2017), corroborando as informações encontradas neste estudo.

Os inventários florestais são essenciais para se conhecer uma determinada área, contudo são caracterizados por ser uma atividade extremamente cara e demorada. Os métodos de amostragem devem sempre ser empregados visando à maior precisão e ao menor custo. A obtenção de mapeamentos do estoque de importantes características de florestas, por meio de um número reduzido de levantamentos 
de campo, representa um grande ganho para as ciências florestais, uma vez que a utilização de imagens de satélites em estudos da cobertura vegetal permite a extração de informações de uma forma rápida e não onerosa (OLIVEIRA et al., 2017).

Diante da grande importância do Cerrado, a carência de informações sobre ele é um fator que não só compromete sua conservação e preservação, mas também inviabiliza a utilização de suas riquezas em função do benefício de toda a sociedade. Cabe à ciência florestal testar e desenvolver técnicas, para que informações de qualidade sejam disponibilizadas, a fim de subsidiar políticas de conservação e estratégias sustentáveis de utilização dos recursos naturais. A aplicação de geotecnologias, em estudos na área florestal, tem se mostrado bastante eficientes. As imagens de sensoriamento remoto atuam como uma fonte eficaz de dados auxiliares para as estimativas de variáveis de interesse, e o emprego de métodos geoestatísticos permite a estimativa das variáveis em áreas não amostradas com elevada precisão. Essas duas técnicas aliadas contribuem para a redução de levantamentos de campo e obtenção de informações mais atuais, como comprovado no presente estudo.

\section{CONCLUSÃO}

A metodologia empregada baseou-se em informações dendrométricas obtidas no passado (2005) e dados de sensoriamento remoto coletados para o ano em que se deseja obter a atualização dos estoques (2010). Os modelos se apresentaram satisfatórios, ajustando-se com aderência as áreas, conseguindo estimar com confiabilidade os estoques de volume e carbono, no ano de 2010, para as diferentes fitofisionomias dentro do Cerrado.

Através deste estudo, podemos inferir que mesmo com uma quantidade menor de pontos amostrados em campo, as estimativas se apresentaram confiáveis para as variáveis estudadas, comprovando que o método é capaz de reduzir o número de levantamentos de campo necessário para mapear com segurança o estoque do potencial de volume e carbono no cerrado. 
O mapeamento do potencial produtivo gerado através da krigagem dos dados estimados se mostrou bastante coerente com a realidade das diferentes fitofisionomias que compõe o cerrado, corroborando com outros estudos já realizados.

Concluímos com o presente estudo que os modelos estimaram com segurança os estoques de volume e carbono para o ano de 2010, tornando possível a obtenção de um mapeamento confiável e atualizado desses estoques para as diferentes fitofisionomias dentro do Cerrado em Minas Gerais sem que mais levantamentos de campo fossem realizados.

\section{REFERÊNCIAS}

ACERBI JÚNIOR, F. W. et al. Change detection in Brazilian savannas using semivariograms derived from NDVI images. Ciência e Agrotecnologia, Lavras, v. 39, n. 2, p. 103-109, mar./abr. 2015.

ALMEIDA, A. Q. et al. Relações empíricas entre características dendrométricas da Caatinga brasileira e dados TM Landsat 5. Pesquisa Agropecuária Brasileira, Brasília, DF, v. 49, n. 4, p. 306-315, abr. 2014.

ALVARENGA, L. H. V. et al. Desempenho da estratificação em um fragmento de cerrado stricto sensu utilizando interpolador geoestatístico. Cerne, Lavras, v. 18, n. 4, p. 675-681, 2012.

ALVARES, C. A. et al. Köppen's climate classification map for Brazil. Meteorologische Zeitschrift, Berlin, v. 22, n. 6, p. 711-728, 2013.

AMARO, M. A. O. et al. Estoque volumétrico, de biomassa e de carbono em uma Floresta Estacional Semidecidual em Viçosa, Minas Gerais. Revista Árvore, Viçosa, MG, v. 37, n. 5, p. 849-857, 2013.

BERRA, E. F. et al. Estimativa do volume total de madeira em espécies de eucalipto a partir de imagens de satélite Landsat. Ciência Florestal, Santa Maria, v. 22, n. 4, p. 853-864, 2012.

CARVALHO, A. M. de et al. Decomposição de resíduos vegetais em Latossolo sob cultivo de milho e plantas de cobertura. Revista Brasileira de Ciência do Solo, Viçosa, MG, v. 32, p. 2831-2838, 2008.

GÓMEZ, C. et al. Modeling forest structural parameters in the mediterranean pines of central Spain using QuickBird-2 imagery and Classification and Regression Tree Analysis (CART). Remote Sensing, Basel, v. 4, p. 135-159, 2012.

GUEDES, I. C. de L. et al. Técnicas geoestatísticas e interpoladores espaciais na estratificação de povoamentos de Eucalyptus sp. Ciência Florestal, Santa Maria, v. 22, n. 3, p. 541-550, jul./ set.2012. 
HYYPPA, J. et al. Accuracy comparison of various remote sensing data sources in the retrieval of forest stand attributes. Forest Ecology and Management, [s. I.], v. 128, n. 1/2, p. 109-120, mar. 2000.

JOURNEL, A. G.; HUIJBREGTS, C. J. Mining geostatistics. London: Academic, 1978. 600 p.

KLINK, C. A.; MACHADO, R. B. A conservação do Cerrado brasileiro. Megadiversidade, São Paulo, v. 1, n. 1, p. 147-155, 2005.

MENDONÇA, R. C. et al. Cerrado: ecologia e flora. Brasília, DF: EMBRAPA Informação e Tecnologia, 2008. v. 2.

MIGUEL, E. P. et al. Redes neurais artificiais para a modelagem do volume de madeira e do cerradão com dados de satélite. Pesquisa Agropecuária Brasileira, Brasília, DF, v. 50, n. 9, p. 829-839, set. 2015.

MORAIS, V. A. et al. Carbon and biomass stocks in a fragment of cerradão in Minas Gerais State, Brazil. Cerne, Lavras, v. 19, n. 2, p. 237-245, 2013.

MORAIS, V. A. et al. Influence of diameter measuring height on the adjustment of volume and biomass equations of cerrado in Minas Gerais. Ciência e Agrotecnologia, Lavras, v. 38, n. 3, p. 230-239, maio/jun. 2014.

OLIVEIRA, I. M. S. et al. Análise multitemporal da regeneração natural da candeia após ocorrência de incêndio florestal. Revista Verde de Agroecologia e Desenvolvimento Sustentável, Mossoró, v. 2, p. 192-197, 2017.

REZENDE, A. V. et al. Comparação de modelos matemáticos para estimativa do volume, biomassa e estoque de carbono da vegetação lenhosa de um cerrado sensu stricto em Brasília, DF. Scientia Forestalis, Piracicaba, n. 71, p. 65-76, 2006.

SANTOS, M. M. et al. Estimativa de parâmetros florestais em área de cerrado a partir de imagens do sensor landsat 8. Floresta, Curitiba, v. 47, n. 1, p. 75-84, mar. 2017.

SCHLERF, M.; ATZBERGER, C.; HILL, J. Remote sensing of forest biophysical variables using HyMap imaging spectrometer data. Remote Sensing of Environment, New York, v. 95, p. 177-194, 2005.

SCOLFORO, H. F. et al. Spatial distribution of aboveground carbon stock of the arboreal vegetation in Brazilian biomes of Savanna, Atlantic Forest and Semi- Arid Woodland. PloS One, San Francisco, v. 10, n. 6, p. e0128781, 2015.

SCOLFORO, J. R. S. Inventário florestal de Minas Gerais: equações de volume, peso de matéria seca e carbono para diferentes fisionomias da flora nativa. Lavras: Ed. UFLA, 2008.

SCOLFORO, J. R. S. et al. Volumetria, peso de matéria seca e carbono. In: INVENTÁRIO florestal de Minas Gerais: cerrado, florística, estrutura, diversidade, similaridade, distribuição diamétrica e de altura, volumetria, tendências de crescimento e áreas aptas para manejo florestal. Lavras: Ed. UFLA, 2008. p. 361-438. 
SCOLFORO, J. R. S.; OLIVEIRA, A. D.; CARVALHO L. M. T. Zoneamento ecológico econômico do estado de Minas Gerais. Lavras: UFLA, 2008. 195 p.

SILVA, S. T. et al. Uso de imagens de sensoriamento remoto para estratificação do cerrado em inventários florestais. Pesquisa Florestal Brasileira, Colombo, v. 34, n. 80, p. 337-343, 2014.

SOUZA, A. A.; GALVÃO, L. S.; SANTOS, J. R. Índices de vegetação derivados do sensor Hyperion/ EO-1 para estimativa de parâmetros biofísicos de fitofisionomias de Cerrado. In: SIMPÓSIO BRASILEIRO DE SENSORIAMENTO REMOTO, 14., 2009, Natal. Anais [...]. Natal: [s. n.], 2009. p. 25-30.

WATZLAWICK, L. F. et al. Estoque de biomassa e carbono na Floresta Ombrófila Mista Montana, Paraná. Scientia Forestalis, v. 40, n. 95, p. 353-362, 2012.

YAMAMOTO, J. K.; LANDIM, P. M. B. Geoestatística: conceitos e aplicações. São Paulo: Oficina de Textos, 2013. $215 \mathrm{p}$.

\section{Contribuição de Autoria}

\section{1 - Ivy Mayara Sanches de Oliveira}

Engenheira Florestal, Me., Analista

https://orcid.org/0000-0002-5680-1232 • ivymayara07@gmail.com

Contribuição: Análise Formal

\section{2 - Aliny Aparecida dos Reis}

Engenheira Florestal, Dra., Pesquisadora

https://orcid.org/0000-0002-7115-1485 • alinyreis@hotmail.com

Contribuição: Validação

\section{3 - Eduarda Martiniano de Oliveira Silveira}

Engenheira Florestal, Dra., Pesquisadora

https://orcid.org/0000-0002-1015-4973•dudalavras@hotmail.com

Contribuição: Curadoria de dados

\section{4 - Fausto Weimar Acerbi Júnior}

Engenheiro Florestal, Dr., Professor

https://orcid.org/0000-0002-9553-0148•fausto@ufla.br

Contribuição: Supervisão 


\section{5 - José Marcio de Mello}

Engenheiro Florestal, Dr., Professor

https://orcid.org/0000-0002-0522-5060•josemarcio@ufla.br

Contribuição: Metodologia

\section{Como citar este artigo}

Oliveira, I. M. S.; Reis, A. A.; Silveira, E. M. O.; Acerbi Júnior, F. W.; Mello, J. M. Estoque do potencial produtivo do cerrado utilizando geotecnologias. Ciência Florestal, Santa Maria, v. 31, n. 2, p. 766-785, 2021. DOI 10.5902/1980509838596. Disponível em: https://doi. org/10.5902/1980509838596. Acesso em: xx mês-abreviado 2021. 